\title{
3. The surveys and register data
}

In this chapter, we will present the two surveys that serve as the data material for the book. The two surveys are called "Community conceptions among ethnic and non-ethnic Danes" (Comcon) and "Migrants' attitudes to welfare" (Mifare) and will mainly be referred to using the two abbreviations. Combined the two surveys have migrants living in Denmark originating from 14 different countries. Mifare has migrants from four countries that, at the time, were members of the European Union (EU) in the form of Poland, Romania, Spain, and Great Britain, and six countries outside the EU and Europe in the form of China, Japan, the Philippines, Turkey, Russia, and the United States (US). The Comcon survey includes migrants from Turkey, Pakistan, Lebanon, Iraq, and ex-Yugoslavia. The two surveys thus cover a wide and diverse selection of the world. In addition, both surveys contain a group of Danes that represent the native population. Throughout this chapter, we will introduce the two surveys in more detail, and discuss similarities and dissimilarities between the two surveys. This we will do by going over the most common issues that face surveys targeted at migrants and discuss how the two surveys handled them.

Migrants and their descendants make up an increasingly large part of the population throughout Western Europe. Therefore, more attention is paid to what migrants think about topics like society and the welfare state. These are the kinds of questions that surveys are traditionally good at answering. However, using existing population surveys to study the migrant sub-populations can be problematic. Even if the country of origin is included in the survey, which is not always the case, then the general population survey would make a poor basis for saying something about what migrants think about a given subject. In the Danish case, migrants and descendants combined made up about 14 per cent of the population in 2015. Therefore, following normal sampling logic a random sample of the population should provide us with the same percentage of migrants and descendants. As a result, we would need approximately 7,000 respondents from a general population survey to find around 1,000 migrants and descendants, which is normally the number needed for statistical analysis. Some studies have pooled comparative surveys over several waves to overcome this problem, but this approach comes with other problems (Dinesen, 2012; Reeskens \& van Oorschot, 2015). This issue is further complicated by the fact that migrants are a very heterogeneous group, who in most cases cannot be treated as one, but have to be divided into sub-groups, often based 
on origin country or ethnicity. For this reason, more and more surveys are being targeted at migrants or specific migrant groups (Font \& Méndez, 2013; Hedegaard, 2017), which is also the method used by this book. However, with this targeting comes new issues that we will cover in this chapter.

\section{DEFINING THE TARGET GROUPS}

The first step of making any survey is defining the target group. In this case, it would be migrants or specific national groups. How the groups are defined is determined by both theoretical and practical considerations. Of theoretical considerations, one could imagine that the survey focused on specific national groups, as they represent certain traits in the migrant population. For instance, the Comcon survey selected migrants that represent some of the largest groups of non-Western migrants in Denmark. The purpose of the study was to study the community conceptions of the migrants originating from these countries since the values and socio-cultural integration of these groups looms large in both public and academic debates (Breidahl, 2017; Breidahl, Holtug, $\&$ Kongshøj, 2018). The Mifare survey, on the other hand, more directly focused on socialization and institution experiences, asking questions about how migrants perceive the welfare institution and how attitudes in the origin country might shape this. Therefore, the origin countries were mainly selected based on being present in international comparative surveys, and maximizing on diversity (Hedegaard \& Bekhuis, 2018; Lubbers, Diehl, Kuhn, \& Larsen, 2018). Further, this socialization perspective meant that the migrants who were included were all over the age of 16 when they arrived in Denmark, that is, they were socialized in the country of origin.

Access to data and how migrants are defined is a more practical consideration. In the Danish context most surveys among migrants, including Comcon and Mifare, are sampled with the help of Statistics Denmark. Statistics Denmark runs the Civil Registration System (Det Centrale Personregister or CPR-register in Danish), which since 1968 has registered everybody with permanent residence in Denmark for more than three months. In the case of refugees, CPR-numbers are assigned from the first day of arrival. The CPR-number is used throughout Danish society, for example for bank accounts, housing contracts, and healthcare, which makes it extremely difficult to live without being registered. This gives us a very reliable way to sample migrants and natives, but also means that Statistics Denmark's definitions of migrants and descendants shape the sampling process. Statistics Denmark defines a migrant as a person born abroad, where none of the parents are born in Denmark and hold Danish citizenship. If the person is born abroad but one parent holds Danish citizenship and is born in Denmark, then he or she is classified as Danish. Descendants are defined as persons born in Denmark 
with both parents being migrants as defined above; with the addition that the descendants turn Danish if one of the parents get Danish citizenship (and abandon former citizenship in a different country). In these definitions, some aspect is implicitly included and excluded. The definitions are, for instance, limited to the first generation (migrants) and the second generation (descendants). Further, the status as a migrant or a descendant can change if the migrant or their parents become Danish citizens. This definition seems very inclusive from a citizenship standpoint but also means that some will be included and excluded (Hedegaard, 2017). For instance, some studies of migrants, based on these definitions, have been criticized for selecting out the most well-integrated migrants who managed to become citizens. These definitions vary across countries and therefore we have to be careful when comparing the results to other contexts, as the sampling procedures might differ a lot (Font \& Méndez, 2013).

\section{SAMPLING}

When the target group has been defined, the next step is to sample it. There is a large literature regarding the sampling (Groves, Fowler Jr, Couper, Lepkowski, Singer, \& Tourangeau, 2011), but in relation to surveys among migrants, there are some specific issues. The first issue is whether to sample among migrants in general or among selected migrant groups. If the sample was drawn among all migrants, then the survey would be able to say something about the attitudes of migrants in general in a given country. This would be the "standard approach" in population surveys. Most survey studies of migrants, however, opt for targeting selected migrant groups. This gives the opportunity to translate the surveys and target the questionnaire towards specific groups. The downside of this is that the survey only is valid regarding these groups and not all migrants. Another aspect to consider is whether to include a sample of the majority population, which can serve as a yardstick or a "control group" (see discussion about assimilation in the previous chapter). Both the Comcon survey and the Mifare survey have opted for the solution of targeting a selected number of migrant groups, as well as a sample of native Danes. Denmark Statistics classification of the country of origin was used. This information is based on the birthplace of a person's mother and secondary her citizenship (if the mother's status is unknown, the father is used, if none is available, then the country or origin is classified as the country of birth of the individual). The samples sizes and number of responses are seen in Table 3.1.

Table 3.1 shows the sample size and number of respondents across the origin countries. For the Mifare survey, the goal was to have at least 300 respondents from each group. This was not fully reached for migrants originating from Turkey, Poland, Romania, and the Philippines. In the Comcon 
Table $3.1 \quad$ Sample sizes, number of responses, and response rates in the Mifare and Comcon surveys

\begin{tabular}{|c|c|c|c|}
\hline \multicolumn{4}{|c|}{ Mifare } \\
\hline Origin country & Sample size & Number of responses & Response rate \\
\hline TUR & 1000 & 216 & 22 \\
\hline ROU & 1000 & 277 & 28 \\
\hline PHL & 1000 & 280 & 28 \\
\hline POL & 1000 & 293 & 29 \\
\hline USA & 900 & 310 & 34 \\
\hline ESP & 900 & 339 & 38 \\
\hline $\mathrm{CHN}$ & 1000 & 346 & 35 \\
\hline JPN & 900 & 379 & 42 \\
\hline GBR & 900 & 402 & 45 \\
\hline RUS & 1000 & 408 & 41 \\
\hline DNK & 900 & 397 & 44 \\
\hline Total & 1050 & 3646 & 35 \\
\hline \multicolumn{4}{|c|}{ Comcon } \\
\hline Origin country & Sample size & Number of responses & Response rate \\
\hline YOU & 315 & 129 & 41 \\
\hline IRQ & 320 & 105 & 33 \\
\hline $\mathrm{LBN}^{1}$ & 315 & 82 & 26 \\
\hline PAK & 315 & 110 & 35 \\
\hline TUR & 315 & 92 & 29 \\
\hline DNK & 1990 & 1182 & 60 \\
\hline Total & 3570 & 1703 & 48 \\
\hline
\end{tabular}

Note: $\quad{ }^{1}$ The group from Lebanon also includes stateless Palestinians.

Source: Data collected from the Mifare survey (2018) and Comcon survey (2015).

survey, the goal was to have at least 100 respondents in each of the groups. This was not fully reached. We also see large variances in the response rate across the two surveys. Low response rates are in themselves bad, as it leads to fewer respondents and less statistical power. This is further complicated by the fact that non-responses are not random, meaning that certain groups like the less well educated, men, and newer migrants tend to be among those who participate less in the surveys (Deding, Fridberg, \& Jakobsen, 2008). This could lead to biases in the results if the attitudes of such sub-groups are underreported, and this has been highlighted as an issue in survey studies of migrant groups (Hedegaard, 2017). 
In order to establish more representative results, we construct statistical weights adjusting for non-response in each of the sampled groups. Since our samples are linked to detailed register data, we know how the sample is distributed across the register variables for both respondents and non-respondents. Therefore, we can pinpoint the non-respondents with larger precision than surveys that use simple population weights. To be more specific we use weighting class adjustment (WCA) (Biemer \& Christ, 2008). A set of variables suspected to be related to non-response is chosen. Within the cells produced by all possible combinations of these variables, response propensities may vary. Respondents are then weighted according to the response propensities within each cell. In this case, the weighting variables are age, equivalized disposable income, cohabitation, citizenship, and employment status. Age is divided into three groups (18-30, 31-45, 46-60 years). Equivalized disposable income is also divided into three roughly equal-sized groups based on the variation within the general population in Denmark (below 200,000 DKK, 200-299,000 DKK, above 300,000 DKK). Cohabitation distinguishes between singles and couples (regardless of marriage or not). Citizenship informs us whether people hold Danish citizenship or not. Employment status simply distinguishes between employment and non-employment (based on the main source of income throughout the year). These weighing procedures do not solve all problems with non-response but do bring us close to representative data for the 14 specific groups and the group of natives.

Generally, both surveys had minor non-response issues that were to be expected, but with some variation across nationalities (Bekhuis, Hedegaard, Seibel, \& Degen, 2018; Kongshøj, 2015). Younger, male, single, and non-employed had response propensities that were a little lower. The biggest differences are to be found with regard to citizenship status in the Comcon survey. Weighting generally reduces the share of respondents with Danish citizenship by about $10-20$ per cent, from a small majority to a large minority (except for the Lebanese migrants where a clear majority possess citizenship, see also Chapter 4). In other words, migrants that had achieved Danish citizenship were overrepresented in the survey. Weighting adjusts citizenship status downward with only a few percentage points in the Mifare survey, but here the share with Danish citizenship is also much smaller in general, as shown in Chapter 4.

What differences does weighting make for our survey questions? This depends on two things: First, the degree of non-response bias on the weighting variables (which, as mentioned, were relatively minor except for citizenship in the Comcon survey), and second, the degree to which the socio-demographic weighting variables affect survey attitudes. As subsequent chapters will show, individual differences in socio-demography do not correlate strongly with welfare attitudes and our other dependent variables. These are the reasons 
why weighting in the end seems to make a relative negligible difference. A comparison of the distribution of some of our dependent variables - attitudes towards redistribution, perceived corruption/institutional distrust, and generalized social trust - by each nationality (not shown) reveals that the statistical weights in our case do not substantially change results. At best, the weights only change attitudes and perceptions for some of the nationalities by a few points on the $0-100$ scales we use throughout the book for each dependent variable. These differences are very small, and they are in any case well within the margin of statistical error. Regardless, we will continue to use weights throughout the book as a safeguard for all statistical analyses.

\section{SIMILARITIES AND DIFFERENCES BETWEEN COMCON AND MIFARE}

The Comcon and Mifare surveys differ in three important ways in regard to their populations of migrants. First, Mifare includes everybody over the age of 18 , while Comcon is limited to the 18- to 60-year-olds. Second, Comcon includes both first and second generation of migrants, while Mifare only includes the first generation. Third, Mifare includes migrants who were over the age of 16 when they migrated, and who have been in Denmark for at least one year, while Comcon does not have these requirements. In terms of the samples among natives, there is also a small difference. Mifare has sampled Danes born in Denmark as children of parents with Danish citizenship. Comcon includes a selection of the general population, which therefore also includes some migrants from all over the world. This amounts to 153 of the 1,282 respondents in the Comcon data, around 12 per cent (as the share should be according to Danmarks Statistik (2016)).

To ensure comparability in the analyses, we have made some cuts in the populations. From both surveys, we only included first-generation migrants between 18 and 60 years old. Thus, those older than 60 were excluded from the Mifare survey, and descendants were excluded from the Comcon survey. In the sample that represents the general population in the Comcon survey, we removed the migrants, to ensure comparability to the Mifare survey. We have chosen not to exclude those who were younger than 16 when they migrated or who have been in the country for less than a year from the Comcon survey. Though this creates small differences in the population, excluding on that criteria would eliminate too many respondents from the Comcon survey, rendering statistical analysis impossible for some of the migrant groups. However, to control for this we will include a variable that measures the number of years lived in Denmark in the analysis. For the Mifare survey, there was also a minor reduction in the number of respondents after the survey data were linked to the administrative data. This was due to a clerical error at the agency responsible 
Table 3.2 Sizes of the populations in the two surveys after mutual exclusion rules

\begin{tabular}{lrcr}
\hline & Mifare & & Comcon \\
\hline DNK & 232 & DNK & 809 \\
TUR & 184 & TUR & 119 \\
ROU & 255 & YOU & 97 \\
PHL & 220 & IRQ & 55 \\
POL & 237 & LBN $^{1}$ & 67 \\
USA & 229 & PAK & 61 \\
ESP & 306 & & \\
CHN & 316 & & \\
JPN & 233 & & 1208 \\
GBR & 279 & & \\
RUS & 365 & & \\
\hline Total & 2856 & & \\
\hline
\end{tabular}

Note: Only included are first-generation migrants or Danes, $18-60$ years old. ${ }^{1}$ The group from Lebanon also includes stateless Palestinians.

Source: Data collected from the Mifare survey (2018) and Comcon survey (2015).

for sampling the data. We are unfortunately not able to account for any bias this creates. Table 3.1 outlines the population sizes following the reduction in sizes using the criteria outlined above.

There are differences as well with respect to the data collection methods and translations. The Mifare survey was translated into the language spoken by most people in the origin country and was collected via mail or web. The Comcon survey was collected via telephone interviews or web, and it was therefore not possible to translate it, as it would require the interviewers to speak a number of languages. Experiences from the Mifare survey show that about half of the respondents chose to answer the survey in their native language, but what it exactly did for the response rates is impossible to say. For both surveys, the respondents reviewed a written invitation to participate along with a link and a code for the web-based collection of the survey. Mifare also sent out a set of written questionnaires, in Danish and the native language, along with a return envelope. The Mifare survey also offered an incentive in the form of a voucher for a movie ticket (value 10 euros) to those who participated in the survey. Mifare sent out two reminders, two weeks apart. For the Comcon survey, the reminder consisted of phone calls and phone interviews for those who preferred that. The Comcon data was collected in 2014, while Mifare data was collected in 2015. 


\section{SCALING OF INDEPENDENT VARIABLES AND STATISTICAL METHOD}

The dependent variables that we use throughout the book are all attitudinal survey questions measured on different scales. Generally, most of them follow 1-5 point-scales such as the standard Likert-scale from "Fully agree" to "Fully disagree", while a few others feature 1-4 point-scales with no middle category. In three cases from the Comcon survey - institutional trust, attitudes towards redistribution, and childcare - respondents replied to a $0-10$ scale. To ensure comparability and easy understanding across chapters, all dependent variables are recoded to a $0-100$ scale. For instance, in the case of a 1-5 point-scale, this means that " 1 " becomes " 0 ", " 2 " becomes " 25 ", " 3 " becomes " 50 ", "4" becomes " 75 ", and " 5 " becomes " 100 ". Naturally, readers should be mindful that our reported differences on a $0-100$ scale refer to differences on an original scale with a much lower number of values. In all chapters, we explain our dependent survey questions and original scales.

Methodologically, we utilize linear (OLS) regression throughout all chapters. Unstandardized linear regression coefficients have the clear advantage that they are easy to understand and interpret. For instance, a regression coefficient of 3.5 simply means that a 1-point increase on the scale of the independent variable (examples could be age or citizenship status) is associated with a 3.5-point increase on the $0-100$ scale of the dependent variable. This would imply, for instance, that each additional year on the age-variable or possessing Danish citizenship (since the latter variable only features two categories on a 0-1 scale) correlates with scoring 3.5 points higher on the dependent variable.

The results from our regression models will be presented in figures with coefficient plots, visualizing coefficients and confidence intervals for each nationality. In these models, native Danes will be the reference point, and coefficients for each immigrant group will then show differences relative to native Danes on the $0-100$ scale of the dependent variable. This makes it easy to visually assess differences in attitudes between our various immigrant groups and native Danes (as well as the statistical confidence intervals for the attitudes of each group), and to see how well our models explain these differences on the $0-100$ scale as we include independent variables in the models.

Generally, subsequent chapters will present at least three models with coefficient plots. The first "binary" model simply shows bivariate coefficients showcasing descriptive group differences on the 0-100 scale of the dependent variable without any other variables taken into account. The second "composition model" shows group differences once we control for the register-based, socio-demographic control variables gender, age, income (distinguishing between wages and benefits), education, and civil status (single, ethnically 
homogenous, and mixed couples). The "full model" takes all other, generally survey-based, variables into account, such as national identification, religiosity, language skills, and so on. This makes it possible to see how well each group of variables manage to explain group differences. Depending on specific chapters, other, generally intermediate models between "composition" and "full model" may show the effects of single variables of particular interest in that chapter. This will be explained if relevant.

\section{ESTABLISHING AN EMPIRICAL FOUNDATION}

It is a cumbersome and difficult task to establish data on migrants' welfare attitudes. The chapter has outlined several pitfalls and challenges. The data are far from optimal. We only have data from a single point in time, we can only select the registered migration and we can only study those that answer our surveys. Furthermore, we have pieced data together from two different surveys, which also came with methodological challenges. However, it is not impossible to collect data on welfare attitudes among migrants. We have answers from 3,023 migrants living in Denmark, who fulfilled all our criteria. We also have a data material based on a randomized sampling within a comprehensive register. Non-registered migrants are not included but this is a group that is close to absent among settled migrants due to the widespread use of CPR-numbers throughout Danish society. Furthermore, we have a weighting procedure, which enables us to adjust for biases in none-response among those selected in the sample. It enables us to reduce the potential pitfall of making inferences from data with a strong overrepresentation of the most assimilated migrants. The rich register information we have on the none-response is a unique feature of our data. This is by no means a guarantee for fully representative data but the data are unique by comparative standards in the field, both in terms of the number of origin countries, the number of respondents, and the (register-based) data quality. We have a unique link between the surveys and rich register data, which enable us to know more about the respondents that answered the survey than what is typically the case. For example, we have detailed information about tax payment, income, and use of welfare benefits from the registers, information that is difficult and sometimes impossible to collect in surveys. We also have registered the time of arrival, citizenship status, and many other indicators. Thus, in the two chapters to come, we will use a mix of survey and register information to describe the 14 migrant groups of the book. 